\title{
The Comparison between Isokinetic Knee Muscles Strength in the Ipsilateral and Contralateral Limbs and Correlating with Function of Patients with Stroke
}

\author{
Fayaz Khan ${ }^{1}$ Haris Anjamparuthikal ${ }^{2}$ Mohamed Faisal Chevidikunnan ${ }^{1}$ \\ ${ }^{1}$ Department of Physical Therapy, Faculty of Medical Rehabilitation \\ Science, King Abdulaziz University, Jeddah, Saudi Arabia \\ 2Department of Neurology, Government Medical College, \\ Kottayam, Kerala, India

\begin{abstract}
Address for correspondence Haris Anjamparuthikal, MD, DM, Department of Neurology, Government Medical College, Kottayam, Kerala 686008, India (e-mail: dr_aaharis@yahoo.com).
\end{abstract}

J Neurosci Rural Pract 2019;10:683-689

\begin{abstract}
Keywords

- isokinetic knee muscle strength

- lower limb function

- proprioception

- stroke

Objective The aim of this study is to compare the isokinetic knee muscles peak torque measurements and proprioception between the affected and intact limbs of patients with stroke, in addition to finding the correlation between knee muscles strength and lower limb function.

Methods Twelve patients with stroke (mean age $64.33 \pm 6.140$ years), with 3 to 7 months poststroke who can walk 25 feet independently without using or using assistive devices and full passive range of motion were included in the study. Biodex isokinetic dynamometer was used for measuring isokinetic strength at $90^{\circ} / \mathrm{s}, 120^{\circ} / \mathrm{s}$, and $150^{\circ} / \mathrm{s}$ and isometric strength at $60^{\circ} / \mathrm{s}$ in both flexors and extensors of the knee, whereas proprioception was measured at $45^{\circ} / \mathrm{s}$ knee flexion, all for affected and intact limbs. Functional measurements were assessed using the Fugl-Meyer Assessment for Lower Limb scale and Barthel Index (BI).

Results The differences shown were found to be statistically significant between affected and intact limbs in isokinetic $90^{\circ} / \mathrm{s}$ flexion $(p=0.005)$, extension $(p=0.0013)$, and isometric at $60^{\circ} / \mathrm{s}$ flexion $(p<0.0001)$ knee muscle strengths and also the proprioception $(p=0.05)$. Significant positive correlation was found between isokinetic affected side knee flexion at $90^{\circ} \mathrm{s}(r=0.903)$ with $\mathrm{BI}(r=0.704)$.

Conclusion There is a significant difference in peak torque measurements between affected and normal lower limbs of poststroke patients, as well as a significant correlation between the knee strength and lower limb functions. Furthermore, it can also be concluded that the differences in knee proprioception between the affected and intact limbs were shown to be significant.
\end{abstract}

\section{Introduction}

The decrease in muscle strength is considered as the leading factor causing motor dysfunction in patients with stroke, described as the difficulty in achieving muscle force to its normal levels due to the reduction in the number of motor units after a cerebrovascular lesion. It is documented that these impairments are found in $89 \%$ of patients in the first week, $72 \%$ in the first month, and $61 \%$ at the sixth month of poststroke. ${ }^{1}$
Muscle strength will be decreased by different factors, primary being muscle disorders or secondary due to spasticity or disuse. The primary weakness can happen earlier after hemiplegia due to stroke rather being secondary to disuse. The pattern of distribution of primary weakness depends on difference between muscle groups; it can be variable between upper and lower limbs and also it shows a difference between proximal and distal muscle segments. ${ }^{2}$ The decrease in muscle strength is also accompanied by a decline 
in muscle peak torque, reduction in the force production velocity, fatigability, and an inefficient force production necessary for optimal task performance. ${ }^{3}$

Other problems causing physical impairments include gait disorders, and spasticity on the affected side, in addition to critical psychological and cognitive difficulties. Therefore, this, in turn, may impact their activities of everyday living and may add more difficulties to return to work, which, in turn, decrease self-recline and lead to dependency in lifestyle. ${ }^{4}$

Recent studies have shown that the capacity of achieving basic activities of daily living (ADL) by the first week will be done by only $12 \%$ of survivors after stroke. However, a few months later, 25 to $74 \%$ will be dependent on human support for basic ADL. One of the most important goals of the functional recovery stage is to improve the walking ability. Around two-thirds of patients can resume an independent walking after stroke; however, only $7 \%$ of them can fully return to walk in their community. ${ }^{5}$ Hence, early and reliable detection is a crucial step for making an appropriate and effective treatment plan. ${ }^{6}$

Previous studies have shown a correlation between muscle strength and the ability to perform functional tasks on patients with stroke. ${ }^{7}$ The isokinetic dynamometer is one of the many types of strength and muscle performance testing apparatuses mentioned in research studies. Most researchers have recommended the use of dynamometers for clinical, laboratory, or research purposes. ${ }^{3}$ It is used for evaluating muscle strength, in both fit and diseased individuals, as it allows the measurement of muscle torque at both concentric and eccentric muscle activities when adjusted to a specific angular velocity, which is considered as one of the dominant elements that impact muscle strength. ${ }^{8}$ Therefore, it provides information regarding muscle performance in relation to functional tasks. ${ }^{9}$ Recently, many studies regarding stroke survivors used techniques of maximum strength for assessing functional and motor performances, recovery, and levels of submaximal strength for research purposes. ${ }^{3}$ Previous literature has reported a significant relation in muscle strengths of the affected limbs and their functional activity. ${ }^{8}$

Thus, the objective of this study is to use the isokinetic dynamometer as an objective assessment tool rather than as a training method to establish the difference between isokinetic knee muscles strength in both affected and normal limbs, and also to find out the relationship with the functional knee scores as to understand how much it may affect the lower limb functions. Apart from that, it is also intended to analyze whether the muscle strength in patients with stroke may have an impact on the knee proprioception as well. Hence, we hypothesized that there will be a reduction in the lower limb muscle strength when compared between the affected and unaffected limbs, which, in turn, may affect the lower limb functional activities and knee proprioception.

\section{Methods}

\section{Subjects}

This was a prospective cross-sectional study where 12 chronic stroke patients were enlisted from the Physical
Therapy Department, Faculty of Applied Medical Sciences from January 15, 2017, to April 20, 2017. The time between the onset of stroke and the first test session was determined as 3 months and above. All the patients were undergoing regular outpatient rehabilitation.

Patients who were 3 months after stroke, could walk at least 25 feet independently with or without using assistive devices, ability to extend knee against gravity, and full passive range of motion in the lower limb, and those who had the cognitive capacity to provide consent were included in the study. Patients with any disease that affects muscle strength and intolerance to exercise testing, and those with grade $1+$ or more shown in the modified Ashworth scale for spasticity were excluded from the study.

The ethical committee of the Faculty of Applied Medical Sciences, King Abdulaziz University, approved the study and written consent from each patient was obtained before they were recruited for the study.

\section{Evaluation}

Initial evaluation using the Fugl-Meyer Assessment for Lower Limb (FMA-LL) and Barthel Index (BI) was performed before the assessment with isokinetic dynamometer.

\section{Isokinetic Dynamometer Assessment}

Knee muscles strength was measured using the Biodex Isokinetic Dynamometer by Biodex Medical System, 2011, Shirley, New York, United States. After the steps of isokinetic testing were explained to provide appropriate orientation with the machine, patients were seated on the chair attached to dynamometer, with firm support to the back. Stabilization was provided across the shoulders, waist, and thigh with straps that are being tested. Patients were asked to keep the arms folded to reduce the involvement of upper extremity. The ankle was fixed proximal to the malleoli by the ankle cuff strap of the lever arm. ${ }^{9}$

\section{Knee Muscle Strength Measurements}

\section{Isokinetic Torques}

Maximal isokinetic knee extension and flexion peak torques were obtained at three concentric velocities, $90^{\circ} / \mathrm{s}, 120^{\circ} \mathrm{s}$, and $150^{\circ} \%$ s. An average of three repetitions was performed for each angular velocity for both affected and intact limbs. Testing started with the $90^{\circ} / \mathrm{s}$ angular velocity and then increased to higher velocities. Before testing, practice trials were given to the patients to familiarize them with the machine.

\section{Maximal Voluntary Force}

Maximal isometric knee extension and flexion forces were obtained at $60^{\circ} / \mathrm{s}$ of knee flexion. For each subject, one set of three repetitions was performed with isometric contractions lasting for 5 seconds. Measurements were obtained for both affected and intact limbs. To prevent fatigue, 10 -second rest was given in between each repetition.

\section{Proprioception}

Proprioception was measured for knee flexion keeping angle at $45^{\circ}$ for both affected and intact limbs. Practice trials were 
given to the subject to ensure the position at $45^{\circ}$ with eyes open followed by the experimental trial with closed eyes.

\section{Data Analysis}

The data were analyzed using statistical software SPSS version 21 (SPSS, Inc., Chicago, Illinois, United States) and Graph Pad version 6 (GraphPad Software Inc., LaJolla, California, United States), significant values were set at $p<0.05$. Normality was tested for the interval/ratio scales with the D'Agostino-Pearson normality test, Shapiro-Wilk normality test, and the Kolmogorov-Smirnov normality test. Parametric analysis tools were used for normally distributed data and nonparametric tools for the not normally distributed data.

\section{Results}

Twelve patients fulfilling the criteria were recruited for the study with a mean age of (64.3 [6.1] years; seven men and five women). Demographic and baseline characteristics of the patients are mentioned in - Table $\mathbf{1}$.

\section{Comparison between Affected and Intact Limbs for Isokinetic Knee Flexion and Extension Peak Torques at $90^{\circ} / \mathrm{s}$}

Using the Mann-Whitney $U$ test, the difference between isokinetic knee flexion peak torques at $90^{\circ} / \mathrm{s}$ was found to be statistically significant at $p=0.005$, with the mean difference being $7.2(1.7)$ and $95 \%$ confidence interval (CI) at 3.57 to 11.01 . The difference between isokinetic knee extension peak torques at $90^{\circ} / \mathrm{s}$ was found to be statistically significant at $p=0.0013$, with the mean difference being 6.6 (1.6) and $95 \%$ CI of 3.18 to 10.07 (-Fig. 1).

\section{Comparison between Affected and Intact Limbs for Isokinetic Knee Flexion and Extension Peak Torques at $120^{\circ} / \mathrm{s}$}

Using unpaired $t$ test, the difference between isokinetic knee flexion peak torques at $120^{\circ} / \mathrm{s}$ was found to be statistically significant at $p<0.0001$, with $95 \% \mathrm{CI}$ ranging between 4.78 and 11.43 and the mean difference being 8.1 (1.6). The difference between isokinetic knee extension peak torques at $120^{\circ} / \mathrm{s}$ was found to be statistically significant at $p=0.0002$, with $95 \% \mathrm{CI}$ between 3.79 and 10.32 and the mean difference of 7.05 (1.5) (-Fig. 1).

\section{Comparison between Affected and Intact Limbs for Isokinetic Knee Flexion and Extension Peak Torques at $150^{\circ} / \mathrm{s}$}

Using the Mann-Whitney $U$ test, the difference between isokinetic knee flexion peak torques at $150^{\circ} / \mathrm{s}$ was found to be statistically significant at $p<0.001$, with $95 \% \mathrm{CI}$ between 4.96 and 11.23 and the mean difference being 8.1 (1.5). The difference between isokinetic knee extension peak torques at $150^{\circ} / \mathrm{s}$ was found to be statistically significant at $p=0.001$, with $95 \% \mathrm{Cl}$ between 4.96 and 11.23 and the mean difference being $6.9(1.7)$ ( - Fig. 1).

Comparison between Affected and Intact Limbs for Knee Isometric Flexion and Extension Peak Torques at $60^{\circ} / \mathrm{s}$ Using unpaired $t$ test, the difference between isometric flexion peak torques was found to be significant at $p<0.0001$,

Table 1 Demographic characteristics of the study subjects

\begin{tabular}{|l|l|l|l|l|l|l|}
\hline Serial number & Age & Sex & $\begin{array}{l}\text { Side of } \\
\text { weakness }\end{array}$ & $\begin{array}{l}\text { Duration after } \\
\text { stroke }\end{array}$ & BI & FMA-LL \\
\hline 1 & 50 & Male & Left & 3 & 55 & 12 \\
\hline 2 & 72 & Male & Left & 6 & 65 & 15 \\
\hline 3 & 67 & Male & Right & 5 & 75 & 24 \\
\hline 4 & 60 & Female & Left & 3 & 80 & 22 \\
\hline 5 & 62 & Female & Right & 4 & 60 & 13 \\
\hline 6 & 65 & Male & Right & 7 & 55 & 11 \\
\hline 7 & 60 & Female & Left & 3 & 50 & 15 \\
\hline 8 & 72 & Male & Right & 3 & 65 & 13 \\
\hline 9 & 70 & Female & Left & 4 & 70 & 16 \\
\hline 10 & 64 & Male & Left & 3 & 75 & 20 \\
\hline 11 & 63 & Female & Left & 4 & 70 & 25 \\
\hline 12 & 67 & Male & Right & 4 & 65 & 22 \\
\hline Mean & 64.33 & 7 male/5 & 5 right/7 left & 4.08 & 65.42 & 17.33 \\
\hline SD & 6.14 & female & & & 1.31 & 9.16 \\
\hline Median & 64.5 & & & 4 & 65 & 4.97 \\
\hline Range & $50-72$ & & & $3-7$ & $50-80$ & 15.50 \\
\hline $95 \%$ Cl & $60.43-68.23$ & & & $5.25-4.92$ & $59.60-71.24$ & $14.15-20.50$ \\
\hline
\end{tabular}

Abbreviations: BI, Barthel Index; Cl, confidence interval; FMA-LL, Fugl-Meyer Assessment for Lower Limb; SD, standard deviation. 


\section{Isokinetic peak torque at different angular velocities}
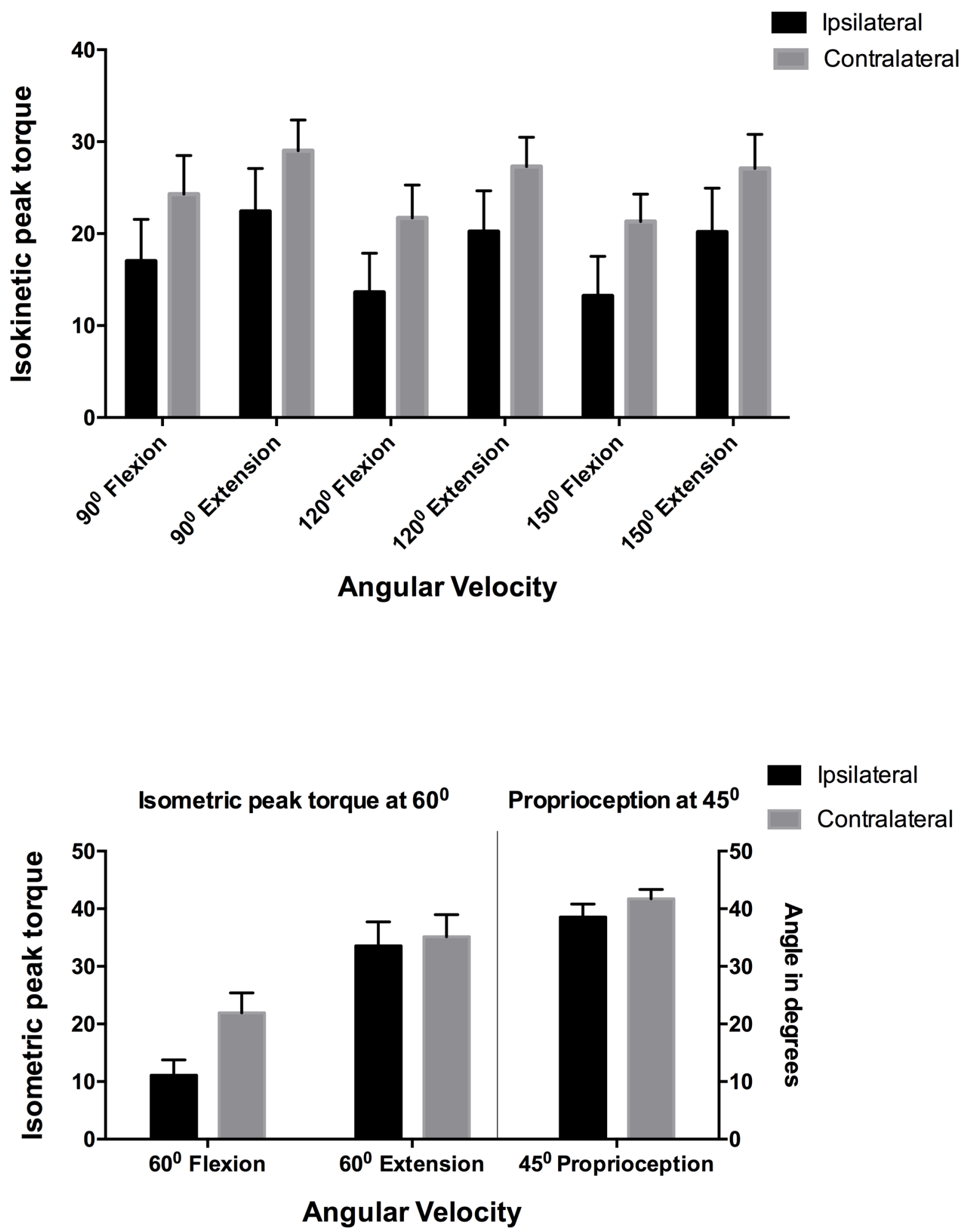

Fig. 1 Isokinetic peak torque at different angular velocities, isometric peak torque at $60^{\circ}$ and proprioception at $45^{\circ}$ between ipsilateral and contralateral lower limbs. 
with $95 \% \mathrm{CI}$ of 8.25 to 13.47 and the mean difference of 8.2 (13.4). The difference between isometric extension peak torques was found not to be significant at $p=0.3531$, with $95 \% \mathrm{CI}$ between -1.83 and 4.93 and the mean difference at 1.5 (1.6) (-Fig. 1).

\section{Comparison between Achieved Proprioception Scores at $45^{\circ} \mathrm{Knee}$ Flexion in Affected and Intact Limbs}

Using unpaired $t$ test, the difference between achieved proprioception scores was found to be significant at $p=0.0006$, with $95 \% \mathrm{CI}$ between 1.51 and 4.80 and the mean difference of 3.1 (0.7) (-Fig. 1).

\section{Correlation between the Fugl-Meyer Assessment for Lower Limb and Barthel Index with isokinetic Flexion at $90^{\circ} / \mathrm{s}$ for the Affected Limb}

Spearman correlation revealed a significant positive correlation between FMA-LL and isokinetic flexion at $90^{\circ} / \mathrm{s}$ $(r=0.903 ; p=0.001 ; 95 \% \mathrm{Cl}=0.673-0.973)$. Similar positive significant correlation was shown between BI and isokinetic flexion at $90^{\circ} / \mathrm{s}(r=0.704 ; p=0.013 ; 95 \% \mathrm{CI}=0.20-0.91)$ (-Fig. 2).

\section{Discussion}

The study showed a significant difference $(p<0.05)$ between isokinetic $90^{\circ} / \mathrm{s}, 120^{\circ} / \mathrm{s}$, and $150^{\circ} / \mathrm{s}$ and isometric $60^{\circ} / \mathrm{s}$ peak torques in flexion and extension of both affected and normal limbs among the poststroke subjects. This finding was in consistent with another similar study, which also proved that there was a significant relation between isokinetic knee flexors and extensors peak torques $(p<0.01)$ at $120^{\circ} / \mathrm{s}$ in the affected and unaffected limbs during concentric contraction. ${ }^{10}$ Studies also documented poor test-retest reliability for the knee flexor torque at $60^{\circ} / \mathrm{s}$ in patients with mild and moderate stroke, which may have been caused by the selected angular velocity.

The reasons for the differences between affected and intact limbs are directly related to the decreased muscle strength and indirectly due to spasticity. In fact, in a previous study, it showed the positive aspect of spasticity in that it does not cause motor disability, which is chiefly caused by the decrease in muscle strength and found no relation between spasticity and muscle strength measured by either isokinetic or isometric measurements. ${ }^{11}$ Muscle weakness, which can be $<60 \%$ of predicted, ${ }^{1}$ eventually leads to a decline in muscle peak torque, the force production velocity, failure to maintain force output, easily fatigued muscles, inadequate force production necessary for task performance, and impaired motor control in both the affected and normal sides with variations between them. ${ }^{6,12}$ Other reasons include contractures, atrophy, disuse or inactivity, alterations in fiber type, and changes in muscle after stroke.

Recent studies have proven that neural changes explained a much greater proportion of poststroke weakness than muscle atrophy. It has been found that neural alternations in the excitability of the cortical, subcortical, and spinal contributions to muscle activation have a relation to insufficient voluntary activation along with alterations in motor unit recruitment. ${ }^{13}$ Even in their studies, Sunnerhagen et $\mathrm{al}^{14}$ and Prado-Medeiros et $\mathrm{al}^{2}$ have stated that there was no muscle atrophy in the thigh muscles in poststroke subjects; however, the knee extensor peak torque was impaired.

There are several studies that prove that the muscle weakness was evident on poststroke subjects' paralyzed limb compared with the normal limb, and moreover, it is also proven that even the intact limb also seems to have reduced strength and peak torque production as compared with the control group. ${ }^{15-18}$ Miller et al ${ }^{19}$ described that the loss of muscle power in the affected limb could be because of the central activation deficits; however, the reason for the bilateral weakness could not be completely explained.

The study by Watanabe et al has used isokinetic dynamometers on mild-to-moderate poststroke hemiparetic patients for the assessment of knee muscle strength and also to measure clinical improvements. ${ }^{10}$ This is in consistent with other studies that reported that all normalized isokinetic strength measures for the flexors of knee and extensors in both the affected and normal limbs were found to be highly reliable. ${ }^{9}$ Kristensen et al also proved that isokinetic dynamometry has generally high reliability to be used in poststroke subjects for documenting the muscle strength. ${ }^{6}$

There was also an important finding suggesting that there is a significant positive correlation existing between FMA-LL and BI with isokinetic flexion at $90 \% \mathrm{~s}$ for the affected limb, which proves that the reduction in the muscle strength or peak torque could ultimately hamper the lower limb function. While a previous study showed that knee muscle strength and gait performance are significantly related $(p \leq 0.01) \cdot{ }^{10}$ Another similar study proved that there is a good correlation between locomotor function and isokinetic peak torque in patients with stroke. ${ }^{20}$ Hunnicutt and Gregory ${ }^{21}$ explained that the correlations of paretic muscle atrophy and bilateral weakness may manifest as asymmetric motor patterns and can affect the gait speeds and for which appropriate gait training interventions are advised. Furthermore, Dawes et al ${ }^{22}$ stated that knee extensor power reduction on poststroke subjects can lead to reduced gait speeds and step lengths. Hence, it can be explained that reduction in muscle strength affects the functional capacity of subjects affected with stroke, which ultimately can lead to an inability to perform routine daily living activities, which can deteriorate the functional independence affecting the quality of life.

The study also found no relation between isometric knee flexion strength at $60^{\circ} / \mathrm{s}$ and proprioception at $45^{\circ}$ for the affected $\operatorname{limb}(r=0.091)$ or between isokinetic knee flexion strength at $90^{\circ} / \mathrm{s}$ and proprioception at $45^{\circ}$ for the affected $\operatorname{limb}(r=-0.21)$. However, there was a significant change in the knee proprioception between the affected and intact lower limbs. In a study, Yang and Kim concluded that knee proprioception level is related to muscle strength in patients with stroke..$^{23}$ In support to their findings in our study, we also could find a difference in knee proprioception between the limbs. Meanwhile, there were no other studies available in the literature to narrate our findings. 


\section{Correlation of FMA-LL Vs Isokinetic $90^{\circ}$ flexion ipsilateral}

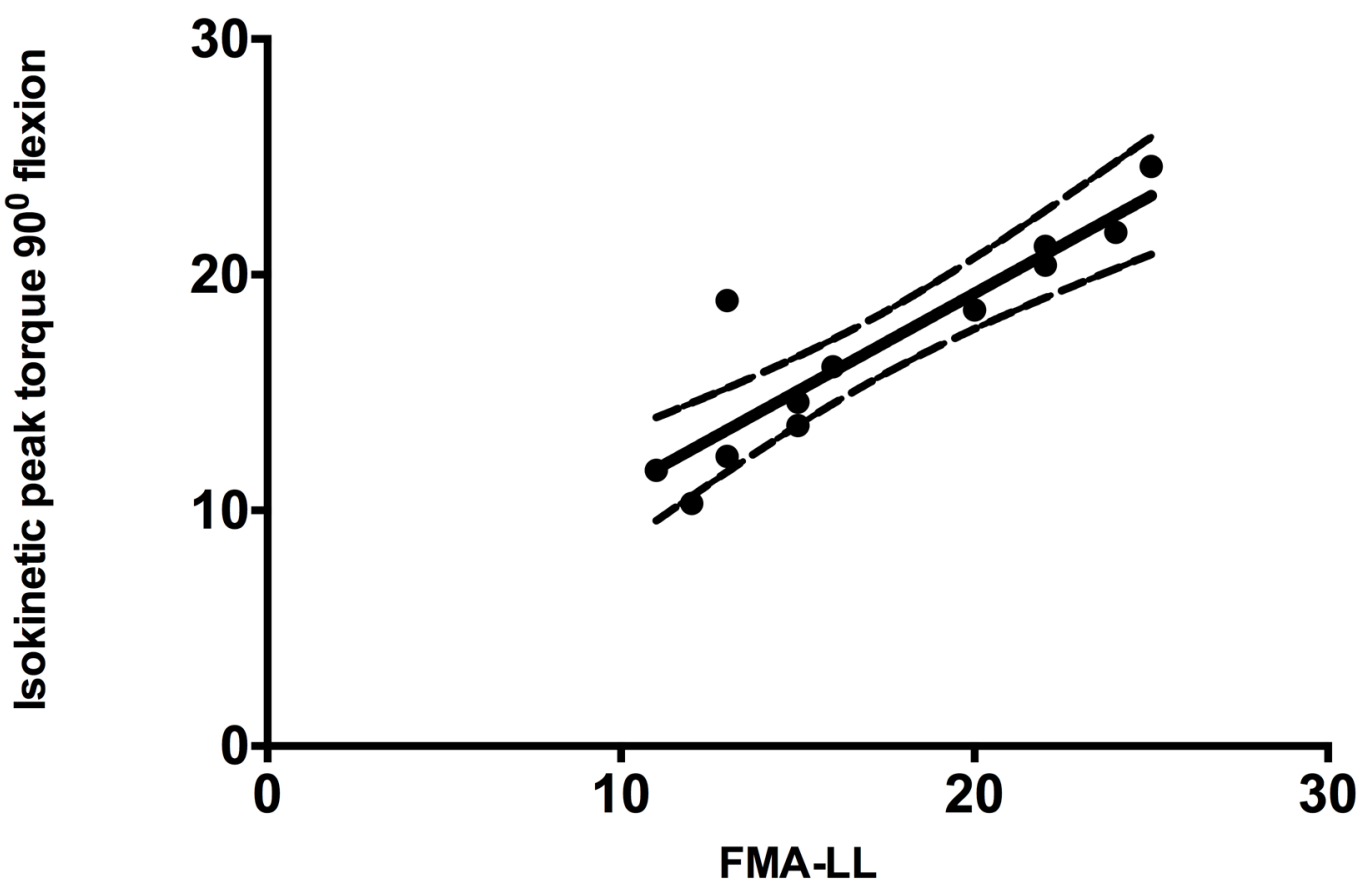

Correlation of BI Vs Isokinetic $90^{\circ}$ flexion ipsilateral

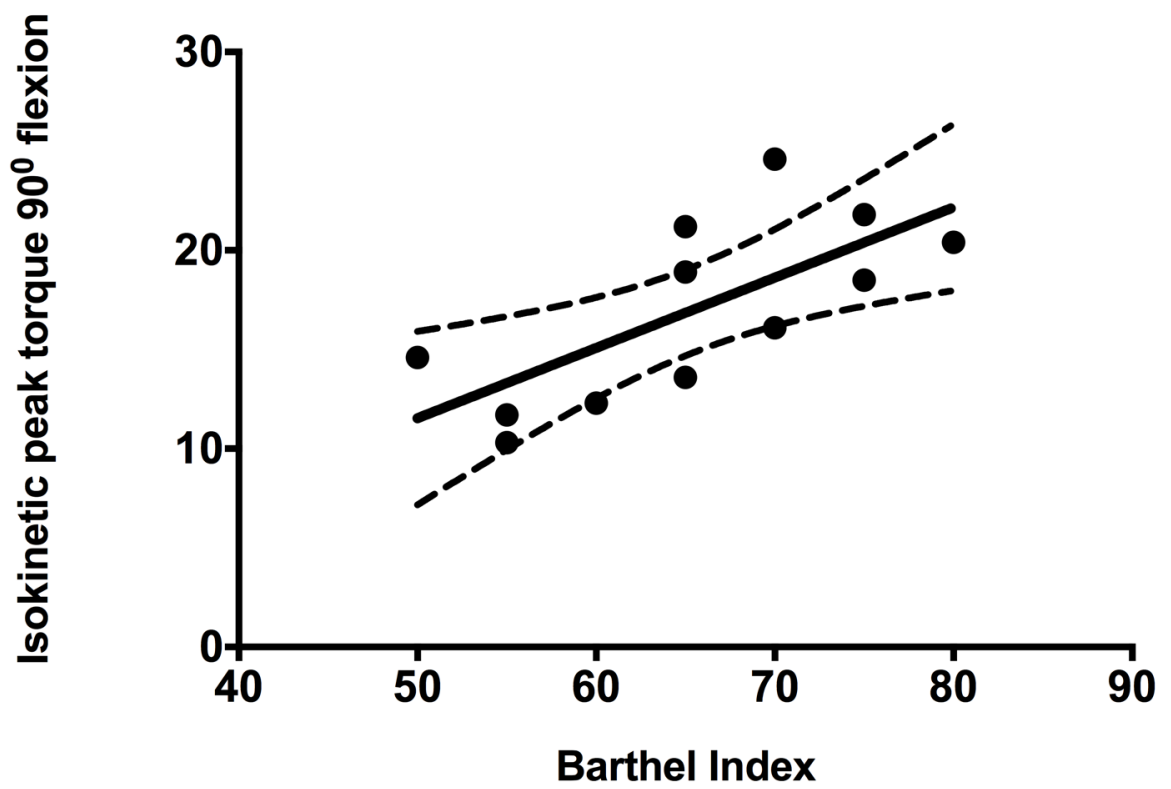

Fig. 2 Correlation of the Fugl-Meyer Assessment for Lower Limb (FMA-LL) and Barthel Index (BI) with isokinetic $90^{\circ}$ flexion of ipsilateral limb. 
With all these various findings, it can be stated and recommended in the clinical practice that, while assessing and treating the patients with stroke, the strength of muscles should be given importance as it is found to be reduced in both affected and normal limbs. The reduction in the muscle strength could affect the lower limb functional activities, and the proprioception also gets altered in the affected limb in patients with stroke.

\section{Conclusion}

The findings from this study suggest that a significant difference in peak torque measurements exists between affected and normal lower limbs of patients with stroke; also, there exists a significant correlation between the strength of knee muscles and lower limb functions. Furthermore, it can also be concluded that the knee proprioception also gets altered in the affected side of the poststroke patients.

\section{Funding}

None.

\section{Conflict of Interest}

None declared.

\section{References}

1 Ivey FM, Prior SJ, Hafer-Macko CE. Katzel LI, Macko RF, Ryan AS. Strength training for skeletal muscle endurance after stroke. J Stroke Cerebrovasc Dis 2017;26(4):787-794

2 Prado-Medeiros CL, Silva MP, Lessi GC, et al. Muscle atrophy and functional deficits of knee extensors and flexors in people with chronic stroke. Phys Ther 2012;92(3):429-439

3 Rabelo M, Nunes GS, da Costa Amante NM, de Noronha M, Fachin-Martins E. Reliability of muscle strength assessment in chronic post-stroke hemiparesis: a systematic review and meta-analysis. Top Stroke Rehabil 2016;23(1):26-36

4 Hung YX, Huang PC, Chen KT, Chu WC. What do stroke patients look for in game-based rehabilitation: a survey study. Medicine (Baltimore) 2016;95(11):e3032

5 Veerbeek JM, van Wegen E, van Peppen R, et al. What is the evidence for physical therapy poststroke? A systematic review and meta-analysis. PLoS One 2014;9(2):e87987

6 Kristensen OH, Stenager E, Dalgas U. Muscle strength and poststroke hemiplegia: a systematic review of muscle strength assessment and muscle strength impairment. Arch Phys Med Rehabil 2017;98(2):368-380

7 Santos AN, Pavão SL, Avila MA, Salvini TF, Rocha NA. Reliability of isokinetic evaluation in passive mode for knee flexors and extensors in healthy children. Braz J Phys Ther 2013;17(2):112-120

8 Chen CL, Chang KJ, Wu PY, Chi CH, Chang ST, Cheng YY. Comparison of the effects between isokinetic and isotonic strength training in subacute stroke patients. J Stroke Cerebrovasc Dis 2015;24(6):1317-1323
9 Li S, Zhuang C, Niu CM, Bao Y, Xie Q, Lan N. Evaluation of functional correlation of task-specific muscle synergies with motor performance in patients poststroke. Front Neurol 2017;8:337

10 Watanabe M, Suzuki M, Sugimura Y, et al. The relationship between bilateral knee muscle strength and gait performance after stroke: the predictive value for gait performance. J Phys Ther Sci 2015;27(10):3227-3232

11 Abdollahi I, Taghizadeh A, Shakeri H, Eivazi M, Jaberzadeh S. The relationship between isokinetic muscle strength and spasticity in the lower limbs of stroke patients. J Bodyw Mov Ther 2015;19(2):284-290

12 Wang W, Li K, Yue S, Yin C, Wei N. Associations between lower-limb muscle activation and knee flexion in post-stroke individuals: a study on the stance-to-swing phases of gait. PLoS One 2017;12(9):e0183865

13 Klein CS, Brooks D, Richardson D, Mcllroy WE, Bayley MT. Voluntary activation failure contributes more to plantar flexor weakness than antagonist coactivation and muscle atrophy in chronic stroke survivors. J Appl Physiol (1985) 2010;109(5):1337-1346

14 Sunnerhagen KS, Svantesson U, Lönn L, Krotkiewski M, Grimby G. Upper motor neuron lesions: their effect on muscle performance and appearance in stroke patients with minor motor impairment. Arch Phys Med Rehabil 1999;80(2):155-161

15 Gerrits KH, Beltman MJ, Koppe PA, et al. Isometric muscle function of knee extensors and the relation with functional performance in patients with stroke. Arch Phys Med Rehabil 2009;90(3):480-487

16 Davies JM, Mayston MJ, Newham DJ. Electrical and mechanical output of the knee muscles during isometric and isokinetic activity in stroke and healthy adults. Disabil Rehabil 1996;18(2):83-90

17 Newham DJ, Hsiao SF. Knee muscle isometric strength, voluntary activation and antagonist co-contraction in the first six months after stroke. Disabil Rehabil 2001;23(9):379-386

18 Harris ML, Polkey MI, Bath PM, Moxham J. Quadriceps muscle weakness following acute hemiplegic stroke. Clin Rehabil 2001;15(3):274-281

19 Miller M, Flansbjer UB, Lexell J. Voluntary activation of the knee extensors in chronic poststroke subjects. Am J Phys Med Rehabil 2009;88(4):286-291

20 Sánchez N, Acosta AM, Lopez-Rosado R, Stienen AH, Dewald JPA. Lower extremity motor impairments in ambulatory chronic hemiparetic stroke: evidence for lower extremity weakness and abnormal muscle and joint torque coupling patterns. Neurorehabil Neural Repair 2017;31(9):814-826

21 Hunnicutt JL, Gregory CM. Skeletal muscle changes following stroke: a systematic review and comparison to healthy individuals. Top Stroke Rehabil 2017;24(6):463-471

22 Dawes H, Smith C, Collett J, et al. A pilot study to investigate explosive leg extensor power and walking performance after stroke. J Sports Sci Med 2005;4(4):556-562

23 Yang JM, Kim SY. Correlation of knee proprioception with muscle strength and spasticity in stroke patients. J Phys Ther Sci 2015;27(9):2705-2708 